\title{
10. Zeolites and Metal-Organic Frameworks as Biomedical Nanodevices
}

\author{
Alejandro Cabrera-García, Zeneida Díaz-Betancor ${ }^{2}$ and \\ Eva Rivero-Buceta ${ }^{1}$ \\ ${ }^{1}$ Instituto de Tecnología Química, Universitat Politècnica de \\ València-Consejo Superior de Investigaciones Científicas, 46022 \\ Valencia, Spain. \\ ${ }^{2}$ Instituto Interuniversitario de Reconocimiento Molecular y \\ Desarrollo Tecnológico, Universitat Politècnica de València, 46022 \\ Valencia, Spain.
}

Keywords: Nanomedicine, cell viability, diagnostic, theranostic, drug delivery, stimuli-responsive, magnetic resonance imaging, combination therapy

\section{From nanotechnology to biomedicine}

Nowadays, there are a wide variety of treatments for all kinds of illnesses. However, many of them are not specifically used to combat the illness for which they were designed. A new field has emerged within nanotechnology to solve this situation. Nanomedicine is responsible for designing, creating and optimizing new pharmacological vectors, which improve the specificity and radius of actuation of current clinical treatments, with the aim of avoiding or reducing side effects during therapy. Nanomedicine is a very broad study area at the present time, mostly focused on the treatment of complicated diseases, such as different types of cancer. For that purpose, the use of nanomaterials takes advantage of the enhanced permeability and retention (EPR) effect, which directs the accumulation of nanomedicines preferentially towards the tumour instead of remaining in plasma or other organs, thus increasing its plasma half-life. Vascular tumours possess poorly aligned, defective endothelial cells with broad fenestrations and no muscular tissue or innervations and a relatively wide lumen. Their receptor function appears altered for vasoactive mediators, especially angiotensin II, and lack functional lymphatics, which greatly contributes to their hyperpermeability. They also show a hyperproduction of vascular mediators 
such as the vascular endothelial growth factor, bradykinin, nitric oxide peroxynitrite, prostaglandins and matrix metalloproteinases [1].

The use of solid drug containers for biomedical purposes requires an accurate control of their dimensions because particles larger than $200 \mathrm{~nm}$ are more easily removed by the splenic filtration system and particles smaller than $10 \mathrm{~nm}$ are likewise cleared through the kidney filtering system. For this reason, the most useful size range is from 10 to $100 \mathrm{~nm}$, which would allow entering the cell by endocytosis, whereas bigger particles or aggregates usually enter the cell by phagocytosis. For in vivo applications of nanoparticles (NPs), their surface needs to be modified in order to protect them from the environment and prolong blood circulation time. Polyethylene glycol (PEG) attachment, mostly known as surface PEGylation, shields the NPs with a hydrophilic coat, which enhances these properties and increases dispersibility whilst avoiding particle aggregation [2].

Porous materials offer many features, which make them especially suitable for biomedical applications. Zeolites and MOFs designed with a biologically friendly composition are potential nanocarriers able to act in different ways to improve human health. Metal-organic frameworks and zeolites can be customized according to the required compositions because of their easy compositional and structural tunability as well as to their size and chemical properties. They possess highly porous structures, which are useful for loading large amounts of therapeutic and imaging agents. The inherent biodegradability of MOFs caused by their relatively labile metal-ligand bonds, in comparison to the high stability of zeolites, increases their applications in this field. So metal-organic frameworks offer a greater variety of biomedical applications than zeolites and that is the reason why we will be citing more examples of MOFs than zeolites throughout the body of the present chapter [3].

\section{Zeolites and MOFs as drug delivery devices}

Drug delivery is the method or process for administering pharmaceutical compounds to achieve a therapeutic effect in humans or animals for the treatment of human diseases [4]. Drug encapsulation and biodegradation are contemplated when developing new drug delivery systems (DDS) and devising potential biomedical applications for MOFs, zeolites and other nanomaterials. Drug loading, which can be carried out by covalent or non-covalent binding methods, are made possible because of their controlrelease properties as well as the highly porous structure of these materials. 
Whereas therapeutic compounds require protection, drugs from vector biodegradation visibly minimize their toxicity and increase their efficiency and half-life in blood. The main objective of drug delivery is to encapsulate molecules of different structures and sizes and release them slowly over several days from the nanocarrier. Nanomaterials are versatile. It is possible to modulate drug delivery by tuning the host-guest interactions through the introduction of various polar or apolar functional groups, or by changing the structure of the solid (interconnectivity, pore size, flexibility) in order to control diffusion through the porous structure. In brief, drug delivery in nanomaterials is controlled by different factors such as the vector degradation in different biological conditions, drug diffusion level through the pores and drug-matrix interactions. The carrier degradation may be affected by complex variables - the carrier solubility, the quality of its surface and others. When the material is very stable in water or other biological environments, drug diffusion mainly depends on the NP porosity and the drug size $[2]$.

In order to demonstrate the utility of synthetic zeolites as drug delivery systems, Rimoli et al. [5] encapsulated the non-steroidal anti-inflammatory drug (NSAID) ketoprofen inside Vegobond ${ }_{13} \mathrm{X}$, a pure zeolite $\mathrm{X}$ with a pore volume of $0.377 \mathrm{~cm}^{3} \mathrm{~g}^{-1}$ and a crystal size of around $2 \mu \mathrm{M}$. Vegobond AX was also used, a mixed phase zeolite with zeolite A in the centre of the crystals and zeolite $\mathrm{X}$, the main component, covering zeolite A. Vegobond AX has a pore volume of $0.374 \mathrm{~cm}^{3} \mathrm{~g}^{-1}$ and the crystal sizes vary from 3 to $8 \mu \mathrm{m}$. Both zeolites accepted the same ketoprofen amount loading of $28.5 \%$. The drug release experiment was carried out at different $\mathrm{pH}$ levels and time periods with the aim of simulating the course of the DDS through the gastrointestinal tract. At an early stage, the loaded zeolites were maintained at $\mathrm{pH} 1$ for an hour and a half, after which the $\mathrm{pH}$ value was increased to 5 and kept at this value for another hour,and the $\mathrm{pH}$ was finally raised to 6.8. The drug platform showed that less than $10 \%$ of the drug was released under acid $\mathrm{pH}$ conditions. However, once the $\mathrm{pH}$ level was increased, the drug loading gradually started to leave the zeolites until its complete delivery. Ketoprofen became thus protected by the zeolite and extended its half-life and bioavailability, which makes it a good choice for treating inflammatory gastrointestinal tract diseases.

Colorectal carcinoma (CRC) is a common type of cancer at present, mostly affecting the male population. In order to fight against CRC, Vilança et al. [6] designed a new drug delivery system hosting the anticancer medicine 5-fluorouracil (5-FU), currently used for the treatment of CRC, stomach, breast, head and neck cancers. 5-FU was loaded into two different 
zeolites - faujasite in the sodium form with two different particle sizes $\mathrm{NaY}$ (70o nm) and nanoNaY (150 nm) and Linde type $\mathrm{L}$ in the potassium form (LTL) with a particle size of $80 \mathrm{~nm}$. Both kinds of zeolites possess a similar pore diameter of around ${ }_{7} \AA ̊$, which allowed 5 -FU loading. The study showed a better effectiveness of 5 -FU loading into $\mathrm{NaY}$ with $71 \%$ than for the nanosized materials with around $55 \%$. The drug release assay was carried out in simulated physiological conditions (phosphate buffer solution, PBS, at $\mathrm{pH}=7.4$ and $37^{\circ} \mathrm{C}$ ) showing $80 \%$ for $\mathrm{NaY}$ and with $90 \%$ released after 48 hours for nanosized materials. In vitro tests were also carried out with these platforms and naked zeolites showed no significant toxicity in the human colorectal carcinoma cell lines tested, HCT-15 and RKO. However, 5-FU loaded zeolites produced a significant decrease in viability in both cell lines and an increase in the effect of 5 -FU within the zeolites when acting freely. In this way, zeolites loaded with 5 -FU are presented as a possible alternative to the administration of this drug in the fight against cancer.

MOFs have also been widely studied as drug carriers. Liu and coworkers [7] designed an interesting system based on hollow nanospheres of the highly stable MOF ZIF-8, which is composed of $\mathrm{Zn}^{2+}$ cations and a 2-methylimidazole ligand. This framework was loaded with $51 \%$ of 5 -FU and finally coated with polymer layers of the developed FA-CHI-5-FAM. The FA-CHI-5-FAM polymer was composed of folic acid (FA) as a targeting molecule, able to bond to the folate receptors overexpressed on many cancer cell surfaces, the chitosan (CHI) linker, which facilitates the polymer formation and its attachment to the nanoparticle surface and, finally, the imaging agent 5 -carboxylfluorescein $(5$-FAM) for monitoring the controlled drug release process. The synthesized nanodevice ZIF-8/5-FU@FA-CHI-5FAM had a size distribution of approximately $400 \mathrm{~nm}$. The lack of toxicity of the non-loaded system was also checked on MGC-803 cells. However, $200 \mu \mathrm{g} \mathrm{mL} \mathrm{m}^{-1}$ of ZIF-8/5-FU@FA-CHI-5-FAM produced the death of $55 \%$ of the cells. Its powerful targeting and signalling effect was also demonstrated by following the strong green fluorescence of the material incubated in MGC-803 cells. Finally, the authors showed the sustained 5-FU release from the nanomaterial in physiological conditions (PBS, $37^{\circ} \mathrm{C}$ ) and in acidic conditions $(\mathrm{pH}=5)$, which favour the dissolution of CHI and ZIF-8. The complete delivery was carried out along 45 and 21 hours respectively. Finally, ZIF-8/5-FU@FA-CHI-5-FAM was presented as a very useful high-loading drug carrier for killing cancer cells controllably.

Here, we give a brief description of another kind of trigger mechanism in a combined therapy system, which we will delve into further in this chapter. It is about directing the drug release through $\mathrm{pH}$ and temperature. 


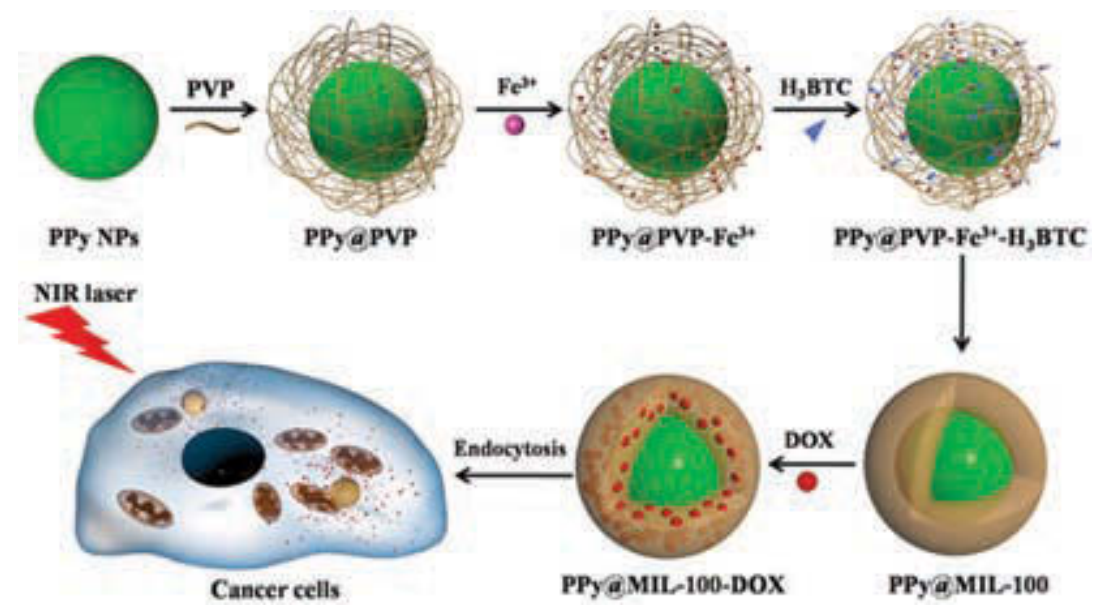

Figure 1. Schematic illustration of multifunctional NPs consisting of a PPy core and a mesoporous MIL-100 shell, designed for simultaneous PTT and chemotherapy of cancer cells. Reprinted with permission from ref. 8. Copyright 2016 American Chemical Society.

In this field, Zhang and coworkers [8] have engineered core-shell nanoparticles composed of organic polypyrrole nanospheres (PPy) in its core, later coated with poly(vinylpyrrolidone) (PVP). This polymer layer made it easier to build a mesoporous MOF shell based on MIL-101(Fe), thus facilitating the coupling between iron (III) cations, bezene-1,3,5-trycarboxylate (BTC) ligands and the PPy NPs surface. The high porosity of MIL-101(Fe) shell was also taken advantage of, in order to load the anticancer drug doxorubicin hydrochloride (DOX). PPy@MIL-10o-DOX NPs of around $107 \mathrm{~nm}$ in size, which were able to accept a $12.8 \%$ DOX loading. The PPy core is able to raise its temperature under near-infrared light (NIR) (Figure 1). This property has proved useful in killing cancer tissue and accelerating the drug delivery process. The nanoplatform was able to release $31.9 \%$ of its DOX load at $\mathrm{pH}=7.4$ and $49.1 \%$ at $\mathrm{pH}=5$ after 120 minutes in the dark because of the MIL-101 $(\mathrm{Fe})$ degradation in acidic conditions. However, whenever the system was treated under NIR irradiation for the same amount of time, such release reached $53.2 \%$ at $\mathrm{pH}=7.4$ and $70.4 \%$ at $\mathrm{pH}=5$. These results demonstrate that the rise in temperature facilitates the DOX delivery thanks to the increase in the molecular mobility of the drug and the movement of the crystalline framework. This effect was checked in HeLa cells in an in vitro assay demonstrating that the DOX free system is non-toxic and that the best results were achieved when incubating the HeLa cells with PPy@MIL-10o-DOX NPs following the NIR laser irradiation, which provoked a faster DOX release and the thermal ablation of 
the cancer cells. This combined system brings us a little closer to directed therapy.

\section{Zeolites and metal-organic frameworks in other biomedical modalities}

Zeolites and MOFs can be very useful in other fields of biomedicine thanks to their excellent properties. They can serve as contrast agents in magnetic resonance imaging (MRI) or in other kinds of diagnostic modalities or therapies. Magnetic resonance imaging is one of the most powerful clinical diagnostic tools due to its non-invasive nature, high spatial resolution, reliance on non-radioactive contrast agents (CAs), infinite penetration depth and anatomic resolution. However, the intrinsically low sensitivity of MRI requires the use of CAs, which accelerate the relaxation rates of surrounded water protons and generate an improvement of sensitivity in the obtained signal. Hence, CAs are often administered in high doses. Based on their relaxation mechanisms, CAs can be classified as $T_{1}$-positive agents modifying the longitudinal relaxation rate, which appears enhanced in brightness or positive contrast when computerized, or as $T_{2}$-negative agents affecting the transversal relaxation rate, thus generating a darkening of the obtained image or negative contrast. As regards this property, it is well known that paramagnetic nanomaterials can often work at very low concentration levels with a remarkable enhancement of the MRI signal. $\mathrm{Gd}^{3+}$ and $\mathrm{Fe}^{3+}$ have the ability of increasing the longitudinal $\left(r_{1}\right)$ and transversal $\left(r_{2}\right)$ water proton relaxation rates in human tissues, thus improving clinical MR images. Those cations, as part of a stable framework, avoid the undesirable side effects of soluble chelates and allow large payloads of paramagnetic metal ions. They also extend the half-lives of the circulating plasma and provide good targeting and accumulation at tumour sites [9]. Hatakeyama et al. [10] studied the influence of particle size of two gadolinium MOFs with 1,4-benzenedicarboxylic acid (1,4-BDC) or 1,2,4-benzenetricarboxylic acid (1,2,4-BTC) over the relaxivity of water protons for MRI. Using controlled micelles, they synthesized Gd MOFs in different shapes and sizes, ranging from $24 \mathrm{~nm}$ to larger than $1 \mathrm{mi}-$ cron (Figure 2). Their magnetic properties were also analysed under a $4.7 \mathrm{~T}$ magnetic field. They found that the particles with a greater area available for the interaction of $\mathrm{Gd}^{3+}$ cations with surrounding water molecules produced greater relaxation rates than larger and coarser particles, which had less surface area. Furthermore, the highest longitudinal relaxivity of $83.9 \mathrm{mM}^{-1} \mathrm{~s}^{-1}$ was shown by the Gd MOF with the highest surface area of $4 \cdot 51 \cdot 10^{19} \mathrm{~nm}^{2}$, and 
(a)

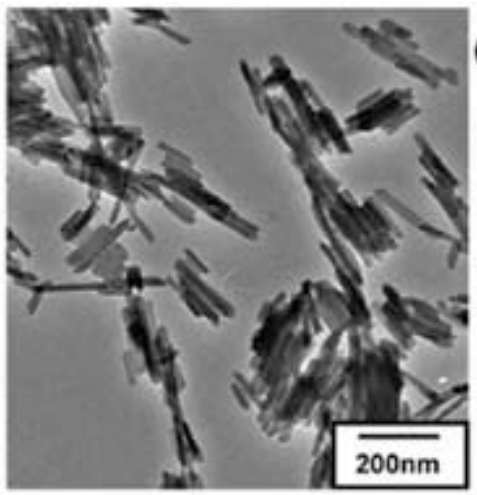

(c)

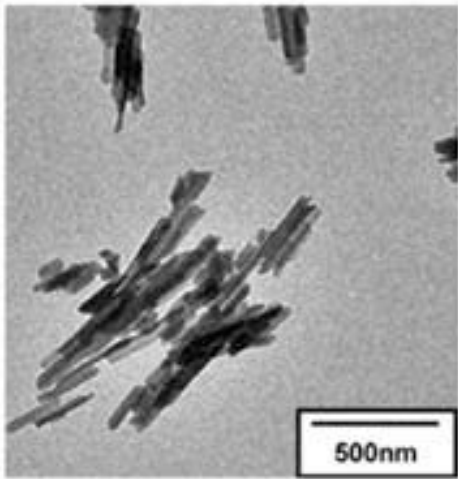

(b)

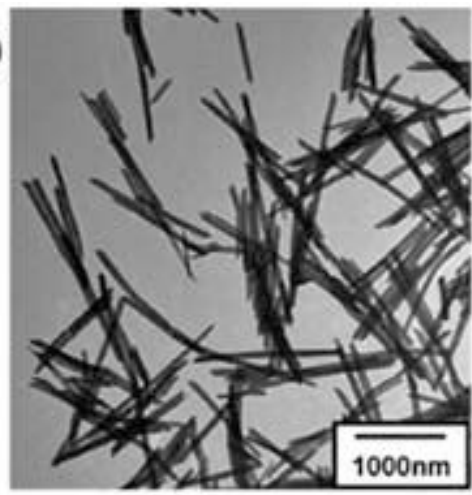

(d)

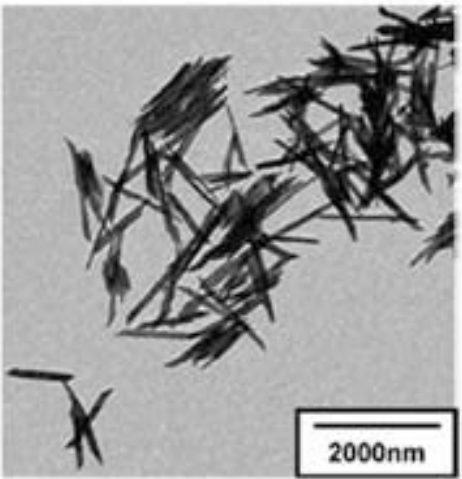

Figure 2. Transmission electron microscopy of 1,4-BDC and $\mathrm{Gd}^{3+}$ MOFs with different shapes and sizes. Reprinted with permission from ref. 10. Copyright 2011 American Chemical Society.

the lowest $r_{1}=17.8 \mathrm{mM}^{-1} \mathrm{~s}^{-1}$ was shown by the largest Gd MOF, with a surface area of $0.893 \cdot 10^{19} \mathrm{~nm}^{2}$. These results demonstrated that the best relaxivity enhancing did not depend on the Gd loaded inside the Gd MOF nanoparticles but on their availability to interact with the surrounding water molecules.

Prussian Blue (PB) and its analogues (PBAs) are a family of hexacyanometallate compounds where two identical or different metal ions are linked by the cyanide ligand, which gives these compounds very interesting properties. Because PB and PBAs are solid frameworks formed by metal ions and organic linkers, they can be considered as the oldest MOFs. Indeed, $\mathrm{PB}$, with a general formula including two different valence iron cations in $\mathrm{Fe}^{\mathrm{III}}{ }_{4}\left[\mathrm{Fe}^{\mathrm{II}}(\mathrm{CN})_{6}\right]_{3} \cdot \mathrm{nH}_{2} \mathrm{O}$, has been used extensively in the pigment industry since 1704 [11]. These compounds are non-toxic and have demonstrated a lot of applications in the biomedical field as biosensors [12], MRI contrast agents [13] and drug delivery devices [14] amongst others. Furthermore, the 
United States Food and Drug Administration (FDA) approved the clinical treatments with the Prussian Blue nanoparticles marketed as Radiogarda$\mathrm{se}^{\mathrm{TM}}$ from HEYL Chemisch-pharmazeutische Fabrik GmbH \& Co. KG as a treatment for internal contamination with radioactive cesium and radioactive or nonradioactive thallium [11]. Recently, Fu et al. [15] reported the application of PB as a photothermal ablation agent for cancer therapy. Photothermal therapy (PTT) is aimed at removing cancer cells through heat generated by photothermal agents, which absorb light energy and convert it into heat. Useful photothermal agents have to work in the near-infrared (NIR) region because the absorption of NIR laser in biological tissues is minimal and the penetration depth is optimal. The authors demonstrated that PB nanocubes with $42 \mathrm{~nm}$ of average diameter were able to increase the temperature in a $500 \mathrm{ppm}$ water suspension to more than $60^{\circ} \mathrm{C}$ when exposed to an $808 \mathrm{~nm}$ laser. They also checked that 3 minutes was enough to reach a temperature of $43 \mathrm{C}$, enough to kill cancer cells. Even though the material suspension suffered some thermal cycles, it remained unaltered and showed good photothermal stability. Finally, the material activity and toxicity of the naked nanovector in HeLa cells was checked to prove that the material did not cause relevant damage in the cell cultures until they were exposed to an $808 \mathrm{~nm}$ laser. The good photothermal activity of the PB nanoparticles was proven because less than $10 \%$ of cell viability was recorded after the treatment with NIR irradiation in the presence of only 16 ppm.

Computed tomography (CT) is a quick and cheap clinical diagnostic tool whereby an X-ray beam is passed through the body of the patient against a movable detector for data collection from various directions and angles. Out of these data, pictures in different directions of the target area will be obtained, which can be used to build up a tomogram in a computer, containing $3 \mathrm{D}$ information about the relevant part of the patient's body. Acting as CAs for CT, the heaviest elements produce a strong scattering of X-rays, so the introduction of these elements will decrease the data collection by the detector in the accumulation areas of these heavy elements. Then, if the heavy elements are well located around the affected tissue, the contrast between normal and diseased tissues will be enhanced in the tomogram, thus contributing a conclusion for the state of the disease [16]. Dekrafft et al. [17] introduced iodine as a heavy element constituent of a MOF able to attenuate X-rays from the irradiated sample, allowing for the construction of images with high spatial resolution. Five nanoscale metal-organic frameworks were synthesized with the bridging ligand 2,3,5,6-tetraiodo-1, 4-benzenedicarboxylic acid $\left(\mathrm{I}_{4}-\mathrm{BDC}\right)$ and $\mathrm{Cu}^{2+}$ or $\mathrm{Zn}^{2+}$ to function as the 
metal cations building up the MOFs. Such NMOFs demonstrated the high $\mathrm{X}$-ray attenuation displayed in phantom studies, which makes them potential CAs for X-ray-based computed tomography. These nanoplatforms showed good biodegradability with a half-life in simulated biological conditions (phosphate buffer saline, $\mathrm{pH}=7.4,37^{\circ} \mathrm{C}$ ) of 1.5 hours. In this period of time prior to its complete dissolution, the new materials can be used as a powerful diagnostic tool in CT.

MOFs can also be used as sensitizing agents in photodynamic therapy (PDT). This treatment uses chemical species like porphyrins, which can be a part of the framework and are able to be excited by light and transfer the energy excess to other molecules like $\mathrm{O}_{2}$ yielding the very unstable form singlet oxygen: ${ }^{1} \mathrm{O}_{2}$. This species can interact with the macromolecules necessary for cell life, killing the cell, or with water, producing reactive oxygen species (ROS), such as superoxide, $\mathrm{O}_{2}^{-}$, hydroxyl radical, $\mathrm{OH}$; or hydrogen peroxide, $\mathrm{H}_{2} \mathrm{O}_{2}$. These very reactive agents can also damage cells. Tissues where singlet oxygen or ROS have been formed by the action of light over a photosensitizer will be killed in a controlled area. The most important limitation of this therapy is that the penetration depth is very short so it is not very useful for deep tumours [16]. Zhang et al. [18] prepared a new system for this purpose by including cationic ruthenium complexes (RCs) able to catalyse ${ }^{1} \mathrm{O}_{2}$ production into the anionic metal-organic framework bio-MOF-1, composed of zinc, adenine (Ad) and biphenyl dicarboxylic acid (BPDC), with the chemical formula $\mathrm{Zn}_{8}(\mathrm{Ad})_{4}(\mathrm{BPDC})_{6} \mathrm{O} \cdot 2 \mathrm{Me}_{2} \mathrm{NH}_{2}$, through an ion-exchange method. Three different sized RCs, $\left(\left[\mathrm{Ru}(\mathrm{bpy})_{3}\right]^{2+}\right.$, bpy = 2,2-bipyridine, the smallest one; $\left[\mathrm{Ru}(\text { phen })_{3}\right]^{2+}$, phen $=1,10$-phenanthroline, and $\left[\mathrm{Ru}(\text { phen })_{2} \text { hipp }\right]^{2+}$, hipp $=2-(1 H$-imidazo[5,5-f][1,10]-phenanthrolin-2-yl)phenol, the largest one) were inserted into the bio-MOF-1, and the composed materials were named bio-MOF-1\&RCs by the authors (Figure 3). Evidently, the RC that entered the framework in the greatest quantity was $\left[\mathrm{Ru}(\mathrm{bpy})_{3}\right]^{2+}$, whereas $\left[\mathrm{Ru}(\mathrm{phen})_{2} \text { hipp }\right]^{2+}$ entered in the lowest amount due to size restrictions. When these materials were irradiated in a single-photon experiment with $490 \mathrm{~nm}$ light, the highest efficiency of ${ }^{1} \mathrm{O}_{2}$ generation was shown by bio-MOF- $1 \&\left[\mathrm{Ru}(\text { phen })_{3}\right]^{2+}$ with a singlet oxygen quantum yield $\left(\varphi_{\Delta}\right)$ of 0.32 . These systems were also assayed for the ${ }^{ } \mathrm{O}_{2}$ generation via two-photon excitation absorption at $800 \mathrm{~nm}$, showing greater singlet oxygen yields thanks to the high electron delocalization of bio-MOF-1 and the energy transfer from the MOF to RCs. For these reasons, this approach was proposed as a PDT sensitizer by the authors.

Another interesting approach to zeolites is their use as hemostatic agents to prevent uncontrolled bleeding after an open injury, which can 


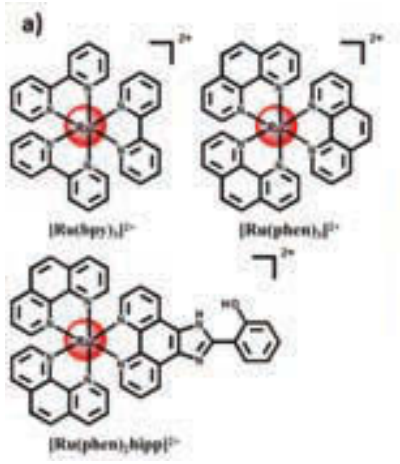

b)

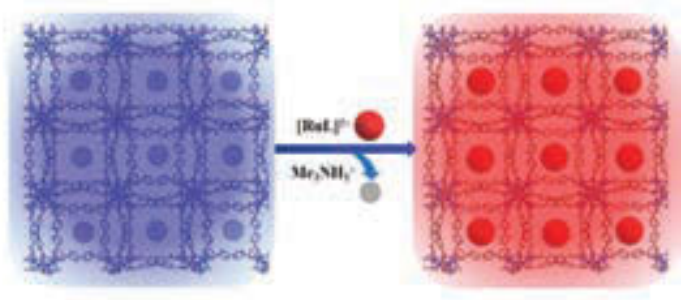

Figure 3. a) Chemical structures of complexes $\left[\mathrm{Ru}(\mathrm{bpyy})_{3}\right]^{2+}$, $\left[\mathrm{Ru}-(\text { phen })_{3}\right]^{2+}$, and $\left[\mathrm{Ru}(\text { phen })_{2} \text { hipp }\right]^{2+}$.

b) Encapsulation of cationic ruthenium(II) complexes $[\mathrm{RuL}]^{2+}$ into the nanospace of bio-MOF-1

( $\mathrm{L}$ stands for 2,2'-bipyridine(bpy), 1,10-phenanthroline(phen), or 2-(1H-imidazo[5,5-f][1,10]-

phenanthrolin-2-yl)phenol(hipp)). Reprinted with permission from ref. 18. Copyright 2016 American Chemical Society.

cause death in many cases. Taeb and coworkers [19] demonstrated that their patent CoolClot, a mixture of bentonite and zeolite minerals, was able to produce an effective decrease in the bleeding time of dogs and in human blood samples - the clotting time in human blood samples was reduced from 253.4 to 143.4 seconds. Zeolites are able to absorb water from the wound tissues by concentrating the biological blood coagulating agents. However, this reaction is exothermic and can cause burns through contact with the affected tissues. The presence of bentonite in CoolClot prevents the rise in temperature and protects the skin in contact with CoolClot from additional damage. Therefore CoolClot stands as a cheap and safe effective hemostatic agent to prevent complications in sports, civil or military accidents.

Due to the extensively adaptable properties of MOFs, they have been proposed for many different sensing applications. Here we will briefly remark on their function as biosensors, that is, a self-sufficient integrated device which is capable of providing specific quantitative or semi-quantitative analytical information using a biological recognition element which is maintained in direct spatial contact with a transduction element [12]. In this sense, Wang et al. [20] developed a label-free colorimetric sensor based on the MOF Fe-MIL-88A, which was built up with iron and fumaric acid. This MOF was able to catalyse the oxidation of 3,3,5,5'-tetramethylbenzidine (TMB) with a very distinct colour change in an aqueous solution. With the introduction of a target biomolecule and its corresponding aptamer, the binding between the target/aptamer and MOF inhibited the catalytic effect of Fe-MIL-88A on TMB, which was used for the sensor design. Their 
sensitive and selective response in complex samples such as human serum was demonstrated by the detection of thrombin, which offered a range of linear detection from o.8 $\mathrm{nM}$ to 8 o $\mathrm{nM}$. Determination could be performed with bare eyes and without absorbance measurements starting at $10 \mathrm{nM}$. This system presents a promising new MOF-based biomolecule detection tool in health care.

\section{Zeolites and MOFs as multifunctional biomedical devices}

Zeolites and MOFs nanocrystals present some valuable properties, which enable them to be nanoplatforms for different kinds of nanomedicinal approaches. Furthermore, due to their compositional and structural tunability, they are able to combine more than one therapeutic or diagnostic modality in the same nanodevice. Here, we combine nanomedicine approaches with various synergistic biomedical effects in the same material. We consider nanomaterials for combination therapy, meaning the use of more than one therapeutic agent or modality on the same platform, multimodal diagnostic imaging and theranostic nanodevices, which integrate diagnosis and disease therapy. This type of nanomedicine brings us closer and closer to personalized medicine.

\section{Combination Therapy}

Combination therapies involve a rational co-delivery of different drugs with synergistic therapeutic effects aimed at improving the single-drug action with different mechanisms of action in the multiple therapeutic modalities and their capacities for hitting multiple targets and overcoming cross-resistance [21]. Many different nanomedical devices have been developed to that purpose. Lin and coworkers [22] prepared a new core-shell $\mathrm{Zn}^{2+}$ bisphosphonate nanoscale coordination polymer (NCP) containing the cisplatin prodrug cis,cis,trans- $\left[\mathrm{Pt}-\left(\mathrm{NH}_{3}\right)_{2} \mathrm{Cl}_{2}\left(\mathrm{OCONHP}(\mathrm{O})(\mathrm{OH})_{2}\right)_{2}\right]$. It was covered with a Polyethylene Glycol (PEG) layer, covalently bounded by a disulfide linkage. These particles were subsequently shielded with cholesterol. The referred nanoplatforms had a spherical shape with a diameter of $105 \cdot 3 \pm 6.2 \mathrm{~nm}$ and were able to combine two therapies - the chemotherapy of cisplatin or cisplatin and gemcitabine and the gene therapy of siRNA by systemic injection. The researchers found a multiple therapeutic vehicle useful for the eradication of cisplatin-resistant ovarian cancer in mouse models. 
Zeolites have also played a biomedical role in this field. De Cola and coworkers [23] designed a zeolitic co-delivery system of DNA and drugs. They used Zeolite L nanocrystals of $5^{\circ} \mathrm{nm}$ as nanocontainers for the blue fluorescent model drug 4,6-diamidino-2-phenylindole (DAPI) for changing the strong negative surface charge due to $\mathrm{OH}$ groups in the zeolite surface. They functionalized the material surface with (3-aminopropyl)triethoxysilane in order to obtain free amino groups on the surface, which were protonated in physiological conditions and allowed an electrostatic anchoring of a DNA oligonucleotide labelled with the red emissive fluorescent dye cyanine5 (Cy5). The authors thereby demonstrated the potential application for this approach in living cells, as it was possible to delay and follow the release of the oligonucleotide as well as the molecules protected inside the nanocontainers.

At times, chemotherapeutics can be combined with photodynamic therapy (PDT), which involves the administration of a tumour-localizing photosensitizer (PS), which is irradiated to generate highly cytotoxic reactive oxygen species (ROS), like singlet oxygen $\left({ }^{1} \mathrm{O}_{2}\right)$, which in turn is able to produce cell apoptosis and necrosis [21]. Here, He et al. [24] combined PDT, chemotherapy and anti-PD-L1 (the PD-1/PD-L1 pathway inhibits immune activation by suppressing effector T-cell function and is upregulated in many tumours to cause apoptosis of tumour-specific cytotoxic T-limphocytes and transmit an antiapoptotic signal to tumour cells) in the same nanomedicine. A NCP was prepared using $\mathrm{Zn}^{2+}$ and oxaliplatin, a prodrug with two phosphate metal donor groups as a core. These nanoparticles were later shielded using phosphate interactions with 1,2-dioleoyl-sn-glycero-3-phosphate (DOPA). The authors took advantage of the hydrophobic interactions between DOPA and other lipid molecules and coated the particles with 1-palmitoyl-2-hydroxy-sn-glycero3-phosphocholine, which had been previously modified with the photosensitizer pyropheophorbide-a via ester bonds. These nanodevices were uniformly spherical with a size of $55 \cdot 3 \pm 0.2 \mathrm{~nm}$ and showed prolonged half-life in blood as well as favourable tumour accumulation after systemic administration. This new system provided a combinational therapy for metastatic colorectal cancer including oxaliplatin as a chemotherapeutic agent and a lipid shell carrying a photosensitizer for PDT and a PD-L1 checkpoint blockade, all three acting as a combined cancer therapy agent, which showed successful results in mice models. PDT can also be combined with checkpoint blockade immunotherapy. Lu et al. [25] have rationally built new nanorod chlorin-based metal-organic framework particles from hafnium and 5,10,15,20-tetra( $p$-benzoato)chlorin. This ligand was chosen 


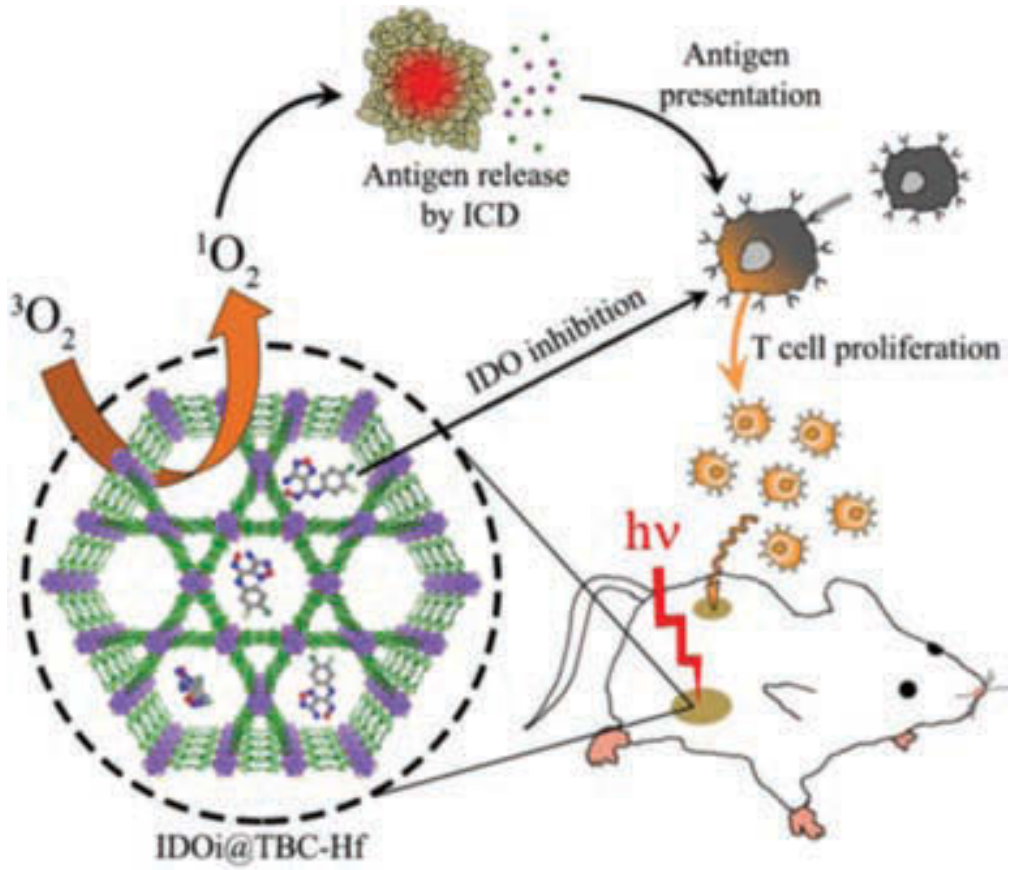

Figure 4. Schematic representation of combined PDT and immunotherapy by IDOi@TBC-Hf. Local injection of IDOi@TBC-Hf and light irradiation generate reactive oxygen species, causing immunogenic cell death (ICD) and releasing tumour-associated antigens, which are presented to T cells. Meanwhile, the IDO inhibitor released from IDOi@TBC-Hf modulates tryptophan/kynurenine catabolism to activate the immunosuppressive tumour microenvironment. The combination of antigen presentation from PDT and checkpoint blockade by IDO inhibition causes T cell proliferation and infiltration, leading not only to eradication of local, treated tumours but also a rejection of distant, untreated tumours. Reprinted with permission from ref. 25. Copyright 2016 American Chemical Society.

on account of its ability to generate highly porous frameworks and because it is an efficient photosensitizer for PDT of cancer. The huge channels allowed loading of small molecules aimed at the inhibition of indoleamine 2, 3-dioxygenase. Such molecules are effective in blocking the expression of this enzyme in cancer processes and enhancing the ROS generation, diffusion and action, especially ${ }^{1} \mathrm{O}_{2}$ after light absorption. The engineered nanomedicine applied to this purpose showed successful results in mice models (Figure 4). This treatment was proposed by the authors as a new way to fight metastatic processes.

Combination therapy also addresses different cancer treatments such as PDT and radiotherapy (RT), which uses X-ray or other sorts of ionizing irradiation to destroy cancer cells. In this sense, Liu and coworkers [26] have designed hafnium MOF nanoparticles. Hafnium is a high-Z element 
functioning as a radio-sensitizer. Photo/auger electrons are generated when the ionizing radiation interacts with these kinds of elements and as a result, the free radicals become able to destroy cancer cells. The ligand was tetrakis(4-carboxyphenyl) porphyrin (TCPP), a photosensitizer for PDT. The material surface was protected with PEG showing an average hydrodynamic diameter of $130 \mathrm{~nm}$ and extremely good stability in physiological conditions. The authors demonstrated the availability of this combined treatment in mice and therefore injected the PEGylated material into tumour-bearing mice, where the material showed long half-life in blood. With no apparent toxicity, the material became rapidly degraded, excreted and showed excellent tumour accumulation levels. They took advantage of this scenario in order to proceed with the RT doses and 8 hours later, so that the tumour oxygenation would be recovered, the tumour was irradiated with $661 \mathrm{~nm}$ light for PDT. Following this combined treatment, they achieved an effective inhibition of the tumour growth.

\section{Multimodal Diagnostic Imaging}

Biomedical imaging is a very important tool in many diagnostics. Due to their compositional tunability, nanoscale metal-organic frameworks can be useful to function as different kinds of contrast agents (CAs) in clinical imaging diagnostic techniques. Furthermore, they can combine the ability to enhance the signal of different imaging techniques in the same material. Rieter et al. [27] chose gadolinium (III) ions as metal nodes to build the framework because its high electronic spin value $(S=7 / 2)$ and slow electronic relaxation rate allows for a decrease in the longitudinal water protons relaxation time, thus enhancing the MRI bright signal around $\mathrm{Gd}^{3^{+}}$. 1,4-benzenedicarboxylate (BDC) was chosen as a binding ligand, yielding $\mathrm{Gd}(\mathrm{BDC})_{1.5}\left(\mathrm{H}_{2} \mathrm{O}\right)_{2}$ nanorods of $100 \mathrm{~nm}$ in length. The authors also doped the NMOFs with $5 \%$ terbium(III) or $5 \%$ europium(III) generating $\mathrm{Gd}_{\text {o.95 }}(\mathrm{BDC})$ $\left(\mathrm{H}_{2} \mathrm{O}\right)_{2}: \mathrm{Eu}_{\text {o.o5 }}$ and $\mathrm{Gd}_{\text {o. } 95}(\mathrm{BDC})\left(\mathrm{H}_{2} \mathrm{O}\right)_{2}: \mathrm{Tb}_{\text {o.o5 }}$ with red and green luminescence respectively. These materials showed large relaxivity values $\left(r_{1}=35.8, r_{2}\right.$ $=55.6 \mathrm{mM}^{-1} \mathrm{~s}^{-1}$ under a 3.0 Tesla magnetic field) and an intense luminescence so they were proposed as a new class of bimodal imaging agents. The same group [28] also chose benzenehexacarboxylate (bhc) ligands because they are able to form stable Gd NMOFs and to carry a high payload of $\mathrm{Gd}^{3+}$ ions. Block-like nanoparticles of $\left[\mathrm{Gd}_{2}(\mathrm{bhc})\left(\mathrm{H}_{2} \mathrm{O}\right)_{6}\right]$ were synthesized in sizes between 25 and $100 \mathrm{~nm}$ which showed low longitudinal relaxivity 


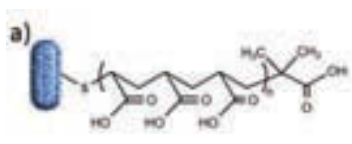

d)

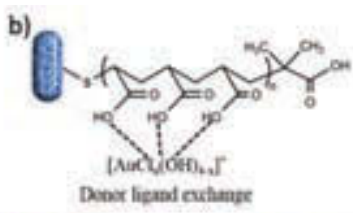

Dener lieasi exhange
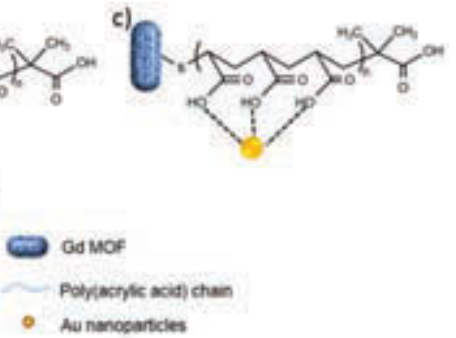

Figure 5. Synthesis layout of GdMOF-PAA-Au nanoparticles a) after deposition of PAA onto GdMOF nanostructures, b) loading of Au ions onto PAA-modified GdMOF nanostructures, followed by c) reduction of the Au ions to produce AuNPs entrapped in the surface immobilized PAA. d) A schematic representation of the structure of hybrid GdMOF-PAA-Au nanostructures. The GdMOF core is shown in blue, the PAA chains as blue chains and the AuNPs in gold. Reprinted with permission from ref. 29. Copyright 2015 American Chemical Society.

$\left(r_{1}=1.5 \mathrm{mM}^{-1} \mathrm{~s}^{-1}\right)$ and very high transversal relaxivity $\left(r_{2}=122.6 \mathrm{mM}^{-1} \mathrm{~s}^{-1}\right)$ under a 9.4 T magnetic field. This large $r_{2}$ rate makes the material a valuable CA for $\mathrm{T}_{2}$-weighted MRI. This material was also doped with $5 \%$ of europium(III) or terbium(III) to make the materials luminescent (green for Tb and red for $\mathrm{Eu}$ ) and useful as CAs for optical imaging in combination with MRI.

Tian et al. [29] developed a new nanocomposite suitable for MRI and $\mathrm{X}$-ray-based computed tomography (CT). CT is a very important diagnostic modality due to its cost effectiveness, wide availability and anatomical imaging ability. It is however, limited to soft tissue resolutions so it would prove more effective when used in combination with MRI as it takes advantage of the Gd-MOFs magnetic properties. Since gold nanoparticles (AuNPs) have a high atomic number and a high X-ray attenuation coefficient, they are very valuable materials for X-ray-based CT imaging. Accordingly, the authors synthesized $\mathrm{Gd}(\mathrm{BDC})_{1.5}\left(\mathrm{H}_{2} \mathrm{O}\right)_{2}$ in nanorod shapes of $155 \mathrm{~nm}$ in length. The vacant orbitals on the $\mathrm{Gd}^{3+}$ ions were used for coordinating the thiol-modified poly(acrylic acid) (PAA) through the thiolate ending groups. The PAA were therefore able to attach $\mathrm{HAuCl}_{4}$ as a $4 \mathrm{~nm}$ AuNPs precursor after the reduction reaction (Figure 5). The nanocomposite GdMOF-PAA-Au was tested for MRI and displayed good brightness enhancement in $T_{1}$-weighted MR images with an $r_{1}$ value of $4.9 \mathrm{mM}^{-1} \mathrm{~s}^{-1}$ at a $4.7 \mathrm{~T}$ magnetic field. The nanocomposite also improved the contrast of CT imaging remarkably even at low gold concentrations and emerged as a promising multimodal diagnostic tool able to enhance the quality of MR and CT images. 


\section{Theranostic Nanoplatforms}

Current trends in nanomedicine are focused on nanoplatforms, which can be loaded with interesting drugs and later tracked by clinical imaging techniques. Theranostics is a word combination of therapy and diagnosis, mostly focused on cancer treatment. These materials open the door to personalized patient treatments because of the possibilities they offer for designing smart systems for controlled drug release. They are also continuously detected from the time they are administered until they are excreted. This enables us to discover the best way to treat different diseases in every different case. Horcajada et al. [30] tested the ability of different biocompatible porous iron carboxylate nanoMOFs (NMOFs) to encapsulate huge amounts of different antitumor, antiviral drugs and cosmetic agents with different polarities, sizes and functional groups. For that purpose, the materials were immersed in concentrated solutions of the product to be entrapped. The authors described the behaviour of the material as 'molecular sponges. Those NMOFs showed a progressive molecular release with no burst effect in a phosphate buffer solution at $37^{\circ} \mathrm{C}$. The effects of the nanoMOF MIL-10o(Fe) loaded with the anti-HIV (anti-Human Immunodeficiency Virus) drug 3'-Azido-2',3'-dideoxythymidine-5'-Triphosphate (AZT-TP) were proved in vitro in human peripheral blood mononuclear cells infected by HIV-1-LAI and approximately $90 \%$ inhibition of HIV replication was achieved. The non-toxicity of the empty material was also tested. The iron cations in the framework structure can also offer a water proton transversal relaxivity rate increase useful for $T_{2}$-weighted MRI diagnosis. In fact, this property was also tested and $r_{2}$ values greater than $50 \mathrm{~s}^{-1} \mathrm{mM}^{-1}$ under a $9.4 \mathrm{~T}$ magnetic field were obtained. PEGylated iron-carboxylate NMOF MIL-88A $\left(r_{2}=95 \mathrm{~s}^{-1} \mathrm{mM}^{-1}\right)$ was used in an in vivo assay as a $T_{2}$ contrast agent for MRI in rats. Darkening in the accumulation tissues (liver and spleen) in the MR images was produced by the CA effect, generating good images for clinical diagnostics.

Similarly, Chen and coworkers [31] developed a new core-shell nanoplatform composed of the Prussian blue analogue $\mathrm{Mn}_{3}\left[\mathrm{Co}(\mathrm{CN})_{6}\right]_{2}$, synthesized through a simple coprecipitation method in which manganese acetate and potassium cobaltocyanide were mixed, and a silica wall generated by tetraethylorthosilicate alkaline polymerization. Finally, they attached $12 \mathrm{~nm}$ silver nanoparticles to the silica surface trough S-Ag coordination bond. For that purpose, $\mathrm{Mn}_{3}\left[\mathrm{Co}(\mathrm{CN})_{6}\right]_{2} @ \mathrm{SiO}_{2}$ nanoparticles were treated with mercaptopropyltrimethoxysilane (MPTMS) dissolved into a silver nitrate solution and reduced with $\mathrm{NaBH}_{4}$. The final material $\mathrm{Mn}_{3}\left[\mathrm{Co}(\mathrm{CN})_{6}\right]_{2} @ \mathrm{SiO}_{2} @ \mathrm{Ag}$ 
presented an average size of $190 \mathrm{~nm}$ and was loaded with doxorubicin (DOX) at a high quantity of $600 \mathrm{mgg}^{-1}$. The release study of DOX was carried out in the dark, where, after $100 \mathrm{~h}$, only $39 \%$ of DOX was released. However, under $808 \mathrm{~nm}$ NIR laser irradiation, the system underwent an additional release of the DOX content of $31 \%$ within the same time interval. This phenomenon was due to Ag nanoparticles converting the laser light irradiation into thermal energy thus accelerating the DOX release. The presence of $\mathrm{Mn}^{2+}$ and $\mathrm{Co}^{3+}$ in the framework is able to accelerate the longitudinal and transversal relaxation rates of the surrounding water protons, which is necessary to achieve efficient CAs for MRI. The recorded values for longitudinal rates were $r_{1}=4.7716 \mathrm{mM}^{-1} \mathrm{~s}^{-1}$ for $\mathrm{Mn}_{3}\left[\mathrm{Co}(\mathrm{CN})_{6}\right]_{2} @ \mathrm{SiO}_{2}$ and $r_{1}=2.4224$ $\mathrm{mM}^{-1} \mathrm{~s}^{-1}$ for $\mathrm{Mn}_{3}\left[\mathrm{Co}(\mathrm{CN})_{6}\right]_{2} @ \mathrm{SiO}_{2} @ \mathrm{Ag}$. For transversal rates, the values were $r_{2}=166.0791 \mathrm{mM}^{-1} \mathrm{~s}^{-1}$ for $\mathrm{Mn}_{3}\left[\mathrm{Co}(\mathrm{CN})_{6}\right]_{2} @ \mathrm{SiO}_{2}$ and $r_{2}=75.5520 \mathrm{mM}^{-1} \mathrm{~s}^{-1}$ for $\mathrm{Mn}_{3}\left[\mathrm{Co}(\mathrm{CN})_{6}\right]_{2} @ \mathrm{SiO}_{2} @ \mathrm{Ag}$ under $3 \mathrm{~T}$ magnetic field. The authors attributed the reduction after $\mathrm{Ag}$ nanoparticles loading to the decrease of the connection area between water and $\mathrm{Mn}_{3}\left[\mathrm{Co}(\mathrm{CN})_{6}\right]_{2}$ inner structure. Besides, $-\mathrm{C} \equiv \mathrm{N}$ ligands in the structure are useful to enhance the fluorescent signal of the material incubated in A549 human lung cells in confocal laser scanning microscopy (CLSM), yielding blue, yellow-green and red fluorescence when the sample is excited with different single-photon laser wavelengths 403, 488 and $543 \mathrm{~nm}$ respectively. Furthermore, this material is also useful to improve two-photon fluorescence imaging (TPFI). This microscopy is more interesting than traditional confocal microscopy because autofluorescence is low and deep penetration and spatial resolution in three dimensions are achieved. The best results were shown by $\mathrm{Mn}_{3}\left[\mathrm{Co}(\mathrm{CN})_{6}\right]_{2} @$ $\mathrm{SiO}_{2} @$ Ag due to the coupling of plasmon resonance of silver nanoparticles and $\mathrm{Mn}_{3}\left[\mathrm{Co}(\mathrm{CN})_{6}\right]_{2}$, which also showed an enhancement in the intensity of TPFI. Finally, the PTT effect was tested showing that $0.05 \mathrm{mgmL}^{-1}$ treated with $808 \mathrm{~nm}$ laser radiation for 10 minutes was enough to reach $43^{\circ} \mathrm{C}$, the critical temperature for this kind of therapy. The material also showed excellent photothermal stability with no change in its morphology. A cell viability study apparently showed no toxicity of the material and $80 \%$ of the HeLa and HepG2 cells survived, even at concentrations of $100 \mu \mathrm{g} \mathrm{mL}$. At a second stage, this study was carried out using NIR irradiation and cell viability was reduced to $16.92 \%$. This approach included the light to heat conversion process, which is able to kill cells through a DOX release effect accelerated by the thermal effect. This material is an ideal example of combination therapy and diagnosis CA nanoplatform. All along the procedure, the progress of the cancer treatment could be monitored through noninvasive clinical diagnosis techniques. 
The same group [32] have also developed a new theranostic nanopatform composed of Prussian blue nanocubes coated with the biodegradable MIL-101(Fe) metal-organic framework, in a dual-MOFs (d-MOFs) system. The nanoagents were prepared to an average size of $190 \mathrm{~nm}$ and loaded with an $85 \%$ of artemisin (ART), a traditional anticancer substance in Chinese medicine. The system is very similar to the one mentioned above - the ART release process was very slow but it accelerated as the $\mathrm{pH}$ level decreased or the temperature increased when the d-MOFs were exposed to the $808 \mathrm{~nm}$ laser light because of the presence of PB mentioned above. The synergy between ART chemotherapy and PTT was successfully tested in vitro in HeLa cells with a cell viability of $20.47 \%$ after the treatment. D-MOFs nanomedicine was also useful as a biological fluorescence marker for multicolour and two-photon bioimaging, as discussed in the previous example - this property was confirmed using human cervical carcinoma HeLa cells. The authors took advantage of the iron cations in the structure in order to shorten water proton relaxation rates $\left(r_{1}=1.313 \mathrm{mM}^{-1} \mathrm{~s}^{-1}, r_{2}=22.258 \mathrm{mM}^{-1} \mathrm{~s}^{-1}\right.$ at $\left.3 \mathrm{~T}\right)$ and checked the MRI enhancement of darkening and brightening in the material accumulation zones after I.V. injection of d-MOFs $(100 \mu \mathrm{L}, 5 \mathrm{mg} / \mathrm{mL})$ into HeLa tumour-bearing mice, which facilitated the mice tumour study. Finally, chemo-photothermal therapy was studied using these d-MOFs, which were able to produce a high tumour inhibition ratio. In view of the results, these systems were positioned as promising nanomedicines for the treatment of cancer in personalized therapies.

\section{Stimuli-Responsive Systems for Drug Delivery}

In recent years, stimuli-responsive nanodevices have emerged as promising drug delivery vehicles (DDSs) due to their capacity for transporting therapeutic agents to the target tissues with no previous release. This effect is possible due to the fact that these vehicles are able to control the release of the drug through a specific stimulus and to minimize undesired side effects. The stimuli responsible for activating the liberation of the therapeutic agent can be classified in a number of ways. In particular, we classify DDSs depending on the stimulus, (endogenous or exogenous) which activates the drug release mechanism [33-35]. An overview of the different types of endogenous/exogenous has been compiled in Figure 6.

Whereas there are various examples of stimuli-responsive MOFs, which can be used for drug delivery in the literature, it is more complicated to find 


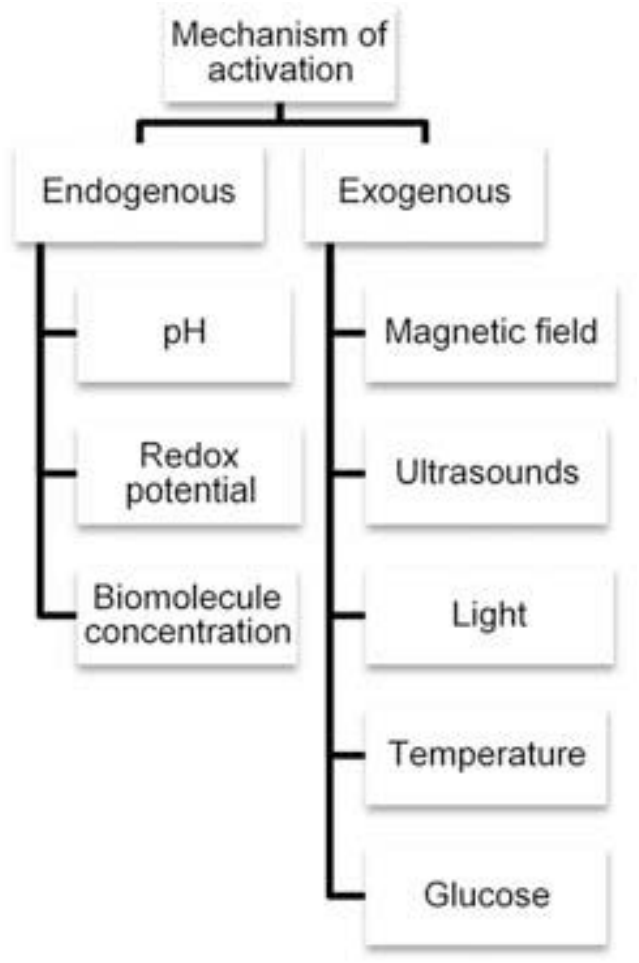

Figure 6. Different types of endogenous/exogenous stimulus.

examples of zeolites. In this section, we summarize the most important systems.

In a pioneering work, Nunzio and coworkers [36] developed a nanoMOF to deliver Topotecan, (TPT) which resembles a 'ship in a bottle' structure (Figure 7). In this work, the authors developed a MIL-10o nanoMOF by coordination self-assembly of $\mathrm{Fe}^{3+}$ octahedral trimers and trimesic acid (BTC) into hybrid supertetrehedra, (ST) which would assemble giving rise to a rigid micromesoporous zeotypic-like structure containing interconnected mesoporous small cages (SC) and large cages (LC) delimited by pentagonal and hexagonal openings respectively. Subsequently, TPT was filled into the pores by means of several consecutive impregnations of MIL-10o in the anticancer agent solutions. The entrapment mechanism could explain the ability of the drug monomers to penetrate into the hexagonal openings but not into the pentagonal windows and the molecules could be loaded in the large cages. Moreover, inside the MOF, the molecules of the monomer were able to aggregate in the large cages through stacking interactions. 


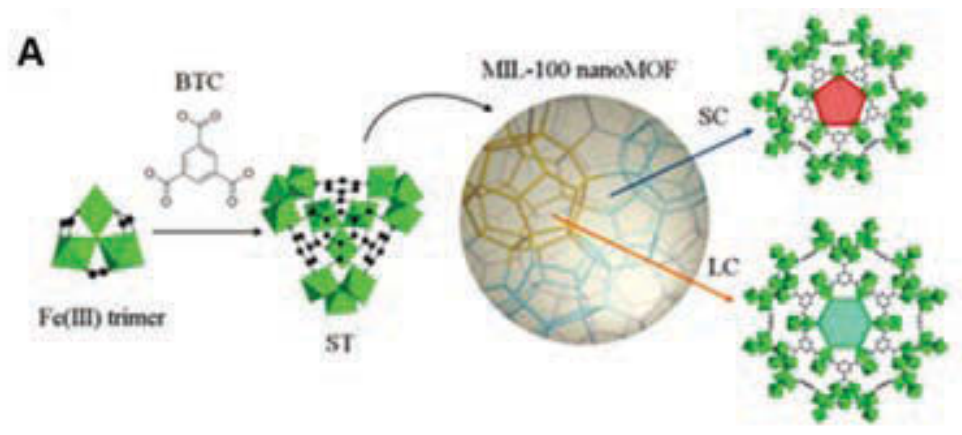

B

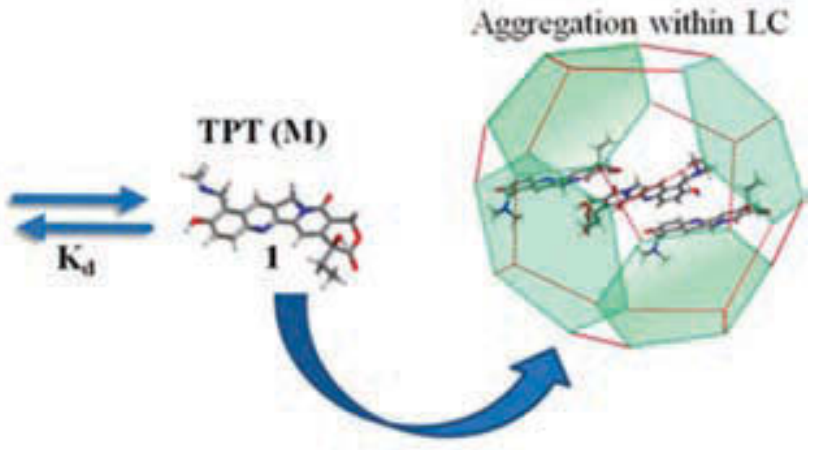

Hexagonal windows crossing

Figure 7. Schematic illustration of the synthesis and structure of MIL-100 nanoMOF structure. A) The nanoMOF possess small cages (SC) and large cages (LC). B) Schematic representation of the hypothesized entrapment mechanism of TPT inside of nanoMOF. Reprinted with permission from ref. 36. Copyright 2014 American Chemical Society.

This new DDS was based upon the one- (OPA) and two-photon absorption (TPA) mechanism to trigger and control the release of the cargo. This concept allows using the near-infrared region (NIR) instead of UV/Vis light as it leads to better tissue penetration because UV/Vis irradiation can damage cells and make it more difficult for the light to penetrate the tissue. In vitro efficacy assays demonstrated that MOFs with no TPT load did not affect the biological activity against A549 alveolar adenocarcinoma and MiaPaCa2 human pancreatic cell lines whereas the nanoMOF loaded with TPT induced cell death with half maximal inhibitory concentration $\left(\mathrm{IC}_{50}\right)$, which were values higher than those observed for the naked drug against the PANCı human pancreatic cell line. One hypothesis to explain the previous effect could point towards the interaction between the nanoMOF and cells, which allows the drug to be transported inside the cells in order to exert the antitumor activity. 


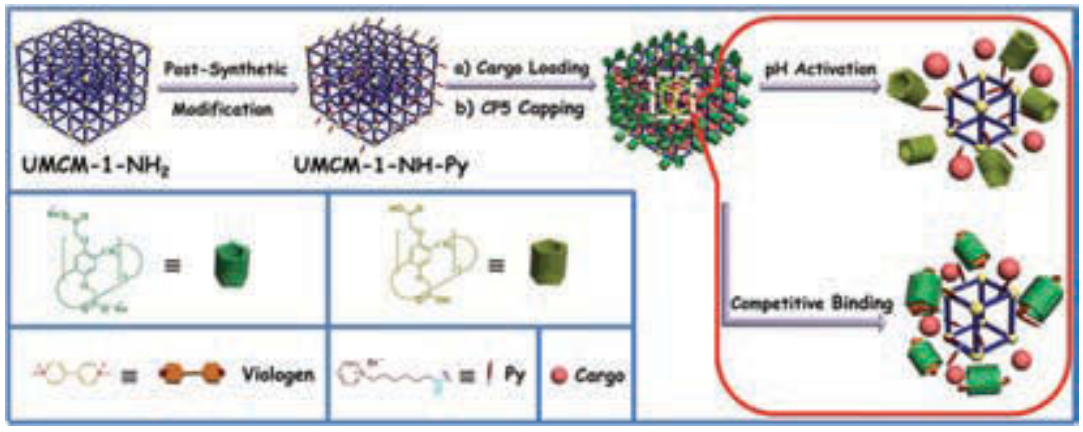

Figure 8. Schematic representation of the stimuli-responsive system based on UMCM-1-NH $\mathrm{Nod}_{2}$ ified with positively-charged pyridinium (Py) stalks and CP5 on the surface. This system, under $\mathrm{pH}$ or competitive binding can regulate the release of cargo molecules. [37] - Published by The Royal Society of Chemistry.

On the other hand, $\mathrm{pH}$-responsive MOFs have been prepared by combining post-synthetic modification (PSM) with stimuli-responsive host-guest chemistry. These MOFs were obtained via post-synthetic modification (PSM), due to the fact that the positively charged pyridinium stalks (py) were tethered onto the surface of UMCM-1- $\mathrm{NH}_{2}$ [37]. Then, rhodamine $6 \mathrm{G}$ was loaded into the nanopores, followed by introduction of the negativelycharged carboxylatepillar[5]arene $\left(\mathrm{CP}_{5}\right)$ via host-guest complexation so as to cap the surface of the nanoMOF (Figure 8). Rh6G release was compared at different $\mathrm{pH}$ values, concluding that nanoMOFs presented a much faster drug release at acid $\mathrm{pH}$ because when the $\mathrm{pH}$ level changed, the pores of the structure opened. Moreover, the study of the release of the cargo through the addition of a competitive binding agent, such as methyl viologen, shows that the release of Rh6G depends on the amount of methyl viologen, which dethreaded the $\mathrm{CP}_{5}$ rings from the Py, causing the drug to release more easily. In vitro cytotoxicity assays in human embryonic kidney cells 293 demonstrated that these materials possessed negligible toxicity.

Recently, a novel water-stable zirconium MOF (Zr-MOF) functionalized with azobenzene moieties was developed as an exclusive structure for on-command drug delivery, which can be activated by different types of external stimuli [38]. To study the release of the cargo, these light-triggered MOFs were loaded with Rhodamine $\mathrm{B}(\mathrm{RhB})$ in the pore interiors and the surface was functionalized with $\beta$-cyclodextrin $(\beta-C D)$ rings, which were threaded onto the azobenzene stalks so as to cap the nanopores and stop the release of the cargo. Upon irradiation with UV light, the isomerization of azobenzene molecule from trans- to cis-form caused the dissociation of the $\beta$-CD ring from the azobenzene stalks. The cis-isomer shows a decreased 
affinity for the $\beta$-CD rings in comparison to trans- isomer, allowing the opening of the nanopores and releasing the cargo. In another experiment, the authors studied the release of the cargo through the addition of a competitive binding agent, such as amantadine. In this case, the RhB release mechanism is based on the competition between amantadine and azobenzene for binding $\beta$-CD rings. Amantadine binds strongly to $\beta$-cyclodextrin, inducing the disruption of the $\beta-C D$ rings from the azobenzene stalks. Moreover, the combination of UV irradiation after the addition of amantadine can improve the amount of RhB released, due to the fact that the trans- to cis- isomerization impels an extra discharge of RhB outwards of the nanopores.

\section{Remarks on the Future}

MOFs are hybrid porous solids formed by the self-assembly between metal ions or clusters and organic ligands. These structures present a number of advantages such as tunable pore size and shape, large surface areas, compositional and structural diversity and biodegradability as a result of the relatively labile metal-ligand bonds. The main characteristic of MOFs is their large pore size, which allows loading a high amount of therapeutic agents. Another advantage of these vehicles is the variety of low-toxic metal ions and organic ligands. All of which are elected to improve biocompatibility and control the stability of the material matrix and the degradation of the structure.

Although more efforts and improvements are needed to test in vitro the behavior of MOFs in physiological conditions and simulated body fluids in order to identify potential hazards associated with their components and it is necessary to evaluate in vivo their degradation mechanisms, biocompatibility and toxicity, their characteristics make them an exceptional tool for future application in the field of nanomedicine.

Zeolites on the other hand, are crystalline inorganic solid materials that possess a three dimensional structure with channels and cavities of uniform size. These structures present a number of properties, such as tunable pore size and shape, large surface areas, bi-modal porosity, diverse morphology and stability in suspension among others. They can serve as transport for therapeutic agents inside the pores or on their surface. Another important characteristic is their low cytotoxicity, which has been reported in the literature. 
In this chapter, we have tried to reflect some of the most interesting characteristics and advantages of zeolites and MOFs, which make them attractive as the object of further investigation in the field of biomedicine on the grounds of the improvements that these kinds of materials can contribute to nanomedicine.

\section{Acknowledgments}

The authors would like to thank the support of Prof. Joan Ripoll-Moragón in developing this chapter. A. Cabrera-García expresses his thankfulness to La Caixa Foundation for granting him a Ph.D. scholarship, Z. Díaz-Betancor is deeply grateful to the Spanish Ministry of Economy and Competitiveness for her scholarship, Dr. E.M. Rivero-Buceta would like to express her gratitude to the Cursol Foundation for her post-doctoral scholarship. Finally, all of us owe a special debt to Dr. Pablo Botella, whose teachings have made it possible for the entire team to develop this project.

\section{References}

[1] Grobmyer, S.R., Moudgil, B.M. (Eds.) Cancer Nanotechnology, Methods and Protocols, ch.3, Springer, 2010.

[2] Giménez-Marqués, M., Hidalgo, T., Serre, C., Horcajada, P. Coord. Chem. Rev. 2016, 307, 342.

[3] He, C., Liu, D., Lin. W. Chem. Rev. 2015, 115, 11079.

[4] Tiwari, G., Tiwari, R., Sriwastawa, B., Bhati, L., Pandey, S., Pandey, P., Bannerjee, S.K. Int. J. Pharm. Investig. 2012, 2, 2.

[5] Rimoli, M.G., Rabaioli, M.R., Melisi, D., Curcio, A., Mondello, S., Mirabelli, R., Abignente, E.J. Biomed. Mater. Res. A 2007, 156.

[6] Vilança, N., Amorim, R., Machado, A.F., Parpot, P., Pereira, M.F.R., Sardo, M., Rocha, J., Fonseca, A.M., Neves, I.C., Baltazar, F. Colloids Surf. B Biointerfaces 2013, 112, 237.

[7] Gao, X., Hai, X., Baigude, H., Guan, W., Liu, Z. Sci. Rep. 2016, 6, 37705.

[8] Zhu, Y.D., Chen, S.P., Zhao, H., Yang, Y., Chen, X.Q., Sun, J., Fan, H.S., Zhang, X.D. Acs Appl. Mater. Interfaces 2016, 8, 34209.

[9] Cabrera-García, A., Vidal-Moya, A., Bernabeu, Á., Pacheco-Torres, J., Checa-Chavarria, E., Fernández, E., Botella, P. Nanomaterials 2016, 6, 109.

[10] Hatakeyama, W., Sanchez, T.J., Rowe, M.D., Serkova, N.J., Liberatore, M.W., Boyes, S.G. ACS Appl. Mater. Interfaces 2011, 3, 1502.

[11] Huang, S.D., Basu, S., Khitrin, A.K., Shokouhimehr, M., Soehnlen. E.S. US Patent 20100215587, 2010.

[12] Tiwari, A., Nordin, A.N. (Eds.) Advanced Biomaterials and Biodevices, ch. 12, John Wiley \& Sons, 2014.

[13] Guari, Y., Larionova, J., Corti, M., Lascialfari, A., Marione, M., Poletti, G., Molvinger, K., Guérin. C. Dalton Trans. 2008, 3658. 
[14] Roy, X., Hui, J.K.H., Rabnawaz, M., Liu, G., MacLachlan, M.J.J. Am. Chem. Soc. 2011, 133, 8420.

[15] Fu, G., Liu, W., Feng, S., Yue, X. Chem. Commun. 2012, 48, 11567.

[16] Dabrowiak, J.C. Metals in Medicine, Ch. 9, John Wiley \& Sons, 2009.

[17] Dekrafft, K.E., Xie, Z.G., Cao, G.H., Tran, S., Ma, L.Q., Zhou, O.Z., Lin, W. Angew. Chem. Int. Ed. 2009, 48, 9901.

[18] Zhang, W., Li, B., Ma, H., Zhang, L., Guan, Y., Zhang, Y., Zhang, X., Jing, P., Yue, S. Acs Appl. Mater. Interfaces 2016, 8, 21465.

[19] Mortazavi, S.M.J., Tavasoli, A., Atefi, M., Tanide, N., Radpey, N., Roshan-shomal, P., Moradi, H., Taeb, S. World J. Emerg. Med. 2013, 4, 123.

[2o] Wang, Y., Zhu, Y., Binyam, A., Liu, M., Wu, Y., Li, F. Biosens. Bioelectron. 2016, 86, 432.

[21] He, C., Lu, J., Lin. W.J. Control. Release 2015, 219, 224.

[22] He, C., Poon, C., Chan, C., Yamada, S.D. Lin, W.J. Am. Chem. Soc. 2016, 138, 6010.

[23] Lülf, H., Bertucci, A., Septiadi, D., Corradini, R., De Cola, L. Chem. Eur.J. 2014, 20, 10900.

[24] He, C., Duan, X., Guo, N., Chan, C., Poon, C., Weichselbaum, R.R., Lin, W. Nat. Commun. 2016, 7, 12499 .

[25] Lu, K., He, C., Guo, N., Chan, C., Ni, K., Weichselbaum, R.R., Lin, W. J. Am. Chem. Soc. 2016, $138,12502$.

[26] Liu, J., Yang, Y., Zhu, W., Yi, X., Dong, Z., Xu, X., Chen, M., Yang, K., Lu, G., Jiang, L., Liu, Z. Biomaterials 2016, 97, 1 .

[27] Rieter, W.J., Taylor, K.M.L., An, H., Lin, W., Lin, W.J. Am. Chem. Soc. 2006, 128, 9024.

[28] Taylor, K.M.L., Jin, A., Lin, W. Angew. Chem. Int. Ed. 2008, 47, 7722.

[29] Tian, C., Zhu, L., Lin, F., Boyes. S.G. ACS Appl. Mater. Interfaces 2015, 7, 17765.

[3o] Horcajada, P., Chalati, T., Serre, C., Gillet, B., Sebrie, C., Baati, T., Eubank, J.F.E., Heurtaux, D., Clayette, P., Kreuz, C., Chang, J.S., Hwang, Y.K., Marsaud, V., Bories, P.N., Cynober, L., Gil, S., Férey, G., Couvreur, P., Gref. R. 2010, 9, 172.

[31] Wang, D., Guo, Z., Zhou, J., Chen, J., Zhao, G., Chen, R., He, M., Liu, Z., Wang, H., Chen, Q. Small 2015, 11, 5956.

[32] Wang, D., Zhou, J., Chen, R., Shi, R., Zhao, G., Xia, G., Li, R., Liu, Z., Tian, J., Wang, H., Guo, Z., Wang, H., Chen, Q. Biomaterials 2016, 100, 27.

[33] Ganta, S., Devalapally, H., Shahiwala, A., Amiji, M.J. Control. Release 2008, 126, 187.

[34] Botella, P., Rivero-Buceta, E.J. Control. Release 2017, 247, 28.

[35] Mura, S., Nicolas, J., Couvreur, P. Nat. Mater. 2013, 12, 991.

[36] Nunzio, M.R., Agostini, V., Cohen, B., Gref, R., Douhal A.J. Med. Chem. 2014, 57, 411.

[37] Tan, L.L, Li, H., Qiu, Y.C., Chen, D.X., Wang, X., Pan, R.Y., Wang, Y., Zhang, S.X.A., Wang, B., Yang, Y.W. Chem. Sci. 2015, 6, 1640.

[38] Meng, X., Gui, B., Yuan, D., Zeller, M., Wang, C. Sci. Adv. 2016, 2, e16oo48o. 